\title{
Lasercooled RaF as a promising candidate to measure molecular parity violation
}

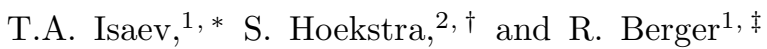 \\ ${ }^{1}$ Clemens-Schöpf Institute, TU Darmstadt, Petersenstr. 22, 64287 Darmstadt, Germany \\ ${ }^{2}$ KVI, University of Groningen, Zernikelaan 25, 9747 AA Groningen, The Netherlands
}

\begin{abstract}
The parameter $W_{\mathrm{a}}$, which characterizes nuclear spin-dependent parity violation effects within the effective molecular spin-rotational Hamiltonian, was computed for the electronic ground state of radium fluoride $(\mathrm{RaF})$ and found to be one of the largest absolute values predicted so far. These calculations were performed with the complex generalised Hartree-Fock method within a two-component (quasi-relativistic) zeroth-order regular approximation framework. Peculiarities of the molecular electronic structure of RaF lead to highly diagonal Franck-Condon matrices between vibrational states of the electronic ground and first excited states, which renders the molecule in principle suitable for direct laser cooling. As a trapped gas of cold molecules offers a superior coherence time, $\mathrm{RaF}$ can be considered a promising candidate for high-precision spectroscopic experiments aimed at the search of molecular parity-violation effects.
\end{abstract}

Diatomic radicals containing heavy nuclei are, together with closed shell chiral molecules with high $Z$ nuclei [1, 2], especially suitable for the search of parity violation effects which depend on the nuclear spin (see review [3]). Up to the present time, the only measured nuclear-spin dependent parity violating (NSDPV) quantity is the anapole moment [4, 5] of the ${ }^{133}$ Cs nucleus [6]. Nevertheless, it was concluded that the result of this experiment is in contradiction with previously obtained restrictions on NSDPV constants (see review [7]), which calls for further attempts to determine NSDPV effects. Currently, an experiment on measurement of the nuclear anapole moment of barium in the barium fluoride $(\mathrm{BaF})$ molecule using a molecular beam is under preparation at Yale University [8]. Here we propose the use of trapped radium fluoride (RaF) molecules as a promising candidate to probe parity violation, which has never been detected in molecules.

In recent years a number of techniques has been developed to prepare, control and cool molecules [9]. A level is now being reached where these techniques can have a significant impact on precision experiments. Stark deceleration, well suited to slow down and trap diatomic polar molecules, has been developed in the last decade [10 12] and the possibility of laser cooling of molecules has only very recently received more attention 13 15. If such cooling methods can be successfully applied to sensitive molecules, a new generation of precision measurements can be done.

As we show in this paper, the RaF molecule appears very well suited for laser cooling methods, because of 1) the highly diagonal Frank-Condon matrix between the electronic ground state and the lowest electronically excited state as well as 2) the facts i) that the corresponding electronic transition frequency is in the visible region with a reasonable lifetime and ii) that isotopes with nuclear spin quantum number $I=1 / 2$ are available. Combined with its large $W_{\mathrm{a}}$, this makes the RaF molecular a prime candidate for a precision experiment probing molecular parity violation.

An experiment with radioactive molecules requires a specialized facility. At the KVI of the University of Groningen the TRI $\mu \mathrm{P}$ facility on the production of radioactive isotopes was commissioned in the year 2007, with the perspective of using these radioactive isotopes in precision experiments to test fundamental symmetries [16]. As the accuracy in a spectroscopic measurement is ultimately limited by the coherence time, there is a clear potential for precision experiments using trapped species. A number of precision experiments using trapped species has been set up, such as $\mathrm{Ra}^{+}$to probe parity violation [17 and Ra atoms for electron electric dipole moment (eEDM) detection 18]. We will discuss the production, deceleration and trapping of $\mathrm{RaF}$ molecules furtheron in this paper.

Calculation of the $W_{\text {a }}$ parameter Previously [19] we utilised the quasi-relativistic two-component (2c) zero order regular approximation (ZORA) for calculating the NSDPV parameter $W_{\mathrm{a}}$ of the effective molecular spinrotational Hamiltonian in a number of heavy-atom diatomic molecules. The corresponding P-odd contribution to this Hamiltonian reads as $W_{\mathrm{a}} k_{A}\left[\vec{\lambda} \times \vec{S}_{\text {eff }}\right] \cdot \vec{I}$ with the effective electron spin operator $\vec{S}_{\text {eff }}$, the nuclear spin operator $\vec{I}$, the vector $\vec{\lambda}$ which points from the heavy nucleus to the light one and the parameter $k_{A}$ of the heavy nucleus 7] that includes the effect of the nuclear anapole moment and the NSD $Z^{0}$ exchange contribution. One may additionally attempt to absorb effects from the nuclearspin independent $Z^{0}$ exchange contribution in $k_{A}$, but as this contribution depends via the hyperfine coupling tensor also on the specific electronic structure, it may appear more canonical to include this contribution in the electronic structure parameter $W_{\mathrm{a}}$. For the time being, however, we have omitted this (supposedly small) additional term.

The results obtained with this approach are at the level of paired generalised Hartree-Fock (paired GHF, for the classification used see review [20]) for off-diagonal matrix elements (MEs) between the degenerate electronic states while diagonal MEs correspond to complex GHF, thereby including spin-polarisation effects. The advantage of our 
approach is that it can be straight-forwardly extended to polyatomic molecules. In the case of diatomic molecules with ${ }^{2} \Sigma$ ground state, we can obtain reliable estimates of the NSDPV effect together with the hyperfine coupling tensor A using ZORA. In our calculations we used a modified version [19, 21, 22] of the TURBOMOLE code [23, 24] with an even-tempered atomic basis set on $\mathrm{Ra}$, a basis set of triple-zeta quality from the TURBOMOLE basis set library augmented with diffuse p- and d-functions on $\mathrm{F}$ atom (see supplementary material at [URL will be inserted by AIP] for basis sets and other parameters of the calculations). A finite spherical Gaussian nucleus model is used and a ZORA model potential as proposed by van Wüllen [25] with additional damping 26] was applied. The equilibrium distance $R_{\mathrm{e}}$ of the electronic ground state was obtained from complex GHFZORA calculations and found to be in good agreement with $R_{\mathrm{e}}$ determined in later four-component (relativistic) Fock space coupled cluster singles and doubles (FSCCSD) calculations. As one can see from the Table I the changes for all parameters are within $6 \%$ when the $R_{\mathrm{e}}$ value is taken either from FS-CCSD or GHF-ZORA calculations. The computed absolute value of the $W_{\text {a }}$ parameter is in the $\mathrm{kHz}$ range and thus one of the largest predicted for diatomic molecules so far. As Ra nuclei have an even number of protons, measurements of $W_{\mathrm{a}}$ in $\mathrm{RaF}$ would complement ideally previous ${ }^{133} \mathrm{Cs}$ experiments on the nuclear anapole moment of nuclei with an odd number of protons. The hyperfine coupling tensor calculated herein for $\mathrm{RaF}$ with accounting for spinpolarisation effects is highly isotropic and can be used, once measured, for estimating the quality of our prediction for $W_{\mathrm{a}}$.

TABLE I: Molecular parameters for the 2c-GHF-ZORA description of the electronic ground state ${ }^{2} \Sigma_{1 / 2}$ of ${ }^{223} \mathrm{RaF}$ and ${ }^{225} \mathrm{RaF}$. The results are given for both $R_{\mathrm{e}}$ taken from the GHF-ZORA $\left(4.40 a_{0}\right)$ and FS-CCSD (4.24 $\left.a_{0}\right)$ calculations. The parallel and perpendicular components of the hyperfine tensor are given in $\mathrm{GHz}, W_{\mathrm{a}}$ in $\mathrm{kHz}$ (with estimated dependence on the Ra mass number being on the order of a few $\mathrm{Hz})$.

\begin{tabular}{l|cc|cc|c}
\multicolumn{4}{|c}{${ }^{223} \mathrm{RaF}$} & \multicolumn{2}{c}{${ }^{225} \mathrm{RaF}$} \\
$R_{\mathrm{e}} / a_{0}$ & $A_{\|} / \mathrm{GHz}$ & $A_{\perp} / \mathrm{GHz}$ & $A_{\|} / \mathrm{GHz}$ & $A_{\perp} / \mathrm{GHz}$ & $W_{\mathrm{a}} / \mathrm{kHz}$ \\
\hline 4.40 & 1.86 & 1.82 & -15.1 & -14.8 & 1.36 \\
& & & & & \\
4.24 & 1.90 & 1.86 & -15.4 & -15.1 & 1.30
\end{tabular}

Besides the high $W_{\mathrm{a}}$ value there is also another peculiarity which makes RaF very attractive for experimental NSDPV search, namely that this molecule appears well suited for being directly cooled with lasers.
Franck-Condon factors and direct laser cooling of molecules One of the major problems in direct laser cooling of molecules is the spontaneous radiative decay into a manifold of rovibrational states not covered by the cooling cycle. Transitions between rotational states are driven by selection rules for vector operators and can be reduced to the two-level case 14], but depopulation of vibrational levels during cooling cycles is the obstacle. In Ref. [13] several molecules were proposed which have highly diagonal overlap matrices between vibrational eigenfunctions of the ground and the first excited electronic states (in terms of the molecular spectroscopy - highly diagonal Franck-Condon matrices). For $10^{4}$ to $10^{5}$ cooling cycles, the probability of the molecule to stay inside the cooling loop has to be greater than 0.9999 with the fourth digit being significant. In practice this means that one has to know FC factors with an accuracy of $0.01 \%$, which is extremely challenging to achieve in modern calculations (although FC factors can in principle be measured with high accuracy). Thus predictions of FC factors with the accuracy required by experimental needs appear practically impossible.

Nevertheless, one can try to narrow down the number of candidate systems for direct cooling with laser based on peculiarities of the molecular electronic structure which renders certain molecules promising. We have analysed the electronic structure of diatomic molecules with one electron over closed shells. Such molecules have large magnetic moments (in comparison with the closed shell systems) which makes them suitable for magnetic trapping. More detailed considerations shall be made in a later article, here we would just like to give the short summary. Highly diagonal FC matrices should be expected when the unpaired electron is making transitions between orbitals that do not contribute to the bonding. Such one-electron wavefunctions can be of the following type: 1) "Electron in lone orbital". This situation is well-known in theoretical chemistry, when, e.g. as a result of $\mathrm{sp}^{n}$ hybridisation, an unpaired electron is directed out of the bonding region. This takes place e.g. in the electronic ground state of $\mathrm{MF}$ and $\mathrm{MH}$, where $\mathrm{M}$ belongs to the group of alkaline earth metals ( $\mathrm{Mg}$ to $\mathrm{Ra}$ ). Two molecules from this series were already used to indicate the perspectives of direct molecular cooling, namely $\mathrm{CaH}$ in Ref. [13] and $\mathrm{SrF}$ in Ref. [14]. 2) "Atom-like electron in molecule". In this case, the outer electron is, due to some reasons (symmetry as a rule), located in a highlysymmetrical state, which has practically atomic nature. Such a situation happens for example in $\mathrm{HI}^{+}$[27], where the unpaired electron resides in an orbital which is mainly of atomic $p_{3 / 2}$ character centered on iodine. In isoelectronic neutral molecules (such as $\mathrm{TeH}$ ) the situation is expected to be the same. 3) In heavy-atom compounds, the valence electron orbital is often quite diffuse, thus the maximum density of the unpaired electron is located further away from the molecular core than in lighter ho- 
mologues. Thus among all possible molecules, those with heavier nuclei appear promising.

The heavy-atom molecules $\mathrm{RaF}, \mathrm{HgH}, \mathrm{HgF}$ etc. which should belong to the first class ("Electron in lone orbital") and $\mathrm{HI}^{+}, \mathrm{PoH}, \mathrm{TeH}$ etc. which should belong to the second class ("Atom-like electron in molecule") are expected to have highly diagonal FC matrices and, thus, appear, in principle, highly suitable candidates for direct cooling of molecules. Unfortunately, the molecules belonging to the second class are not particularly suitable for NSDPV experiments as the unpaired valence electron is in an orbital with high total angular momentum and does not have noticeable spin density in the vicinity of heavy nucleus. As to the first class, the molecules $\mathrm{HgH}$ and $\mathrm{HgF}$ have (as RaF) a ${ }^{2} \Sigma$ ground state and, according to our estimates, even higher $W_{\mathrm{a}}$ values (for $\mathrm{HgH}$ it is $3.3 \mathrm{kHz}$, although we would like to emphasize again that spin-polarisation and other electron correlation effects are not accounted in our $W_{\mathrm{a}}$ estimates, while these effects can well be important). The excitation energy of the first excited state in these molecules, however, is in violet or ultraviolet region (for $\mathrm{HgH} 407 \mathrm{~nm}$, for $\mathrm{HgF} 256$ $\mathrm{nm}$ ) [28]. Especially at $256 \mathrm{~nm}$ it is difficult to produce sufficiently intense laser light. In the series MF, instead, where $\mathrm{M}$ belongs to the group of alkaline earth metals, the excitation energies from the ground to first excited electronic states are in the visible region, so one can expect a similar situation in $\mathrm{RaF}$. This wavelength region is directly accessible using diode lasers.

To substantiate the considerations above, we performed exemplary four-component Fock space (FS) CCSD calculations of the six lowest-lying states of RaF using the DIRAC08 package [29]. The details of the FSCCSD approach are given in the review [30]. Electrons of the $6 \mathrm{~s}, 6 \mathrm{p}, 7 \mathrm{~s}$ shells of $\mathrm{Ra}$ and $2 \mathrm{~s}, 2 \mathrm{p}$ of $\mathrm{F}$ are correlated, the basis sets and other computational parameters can be located in the supplementary material.

The two energetically lowest lying electronic states are identified as ${ }^{2} \Sigma_{1 / 2}$ (ground state) and ${ }^{2} \Pi_{1 / 2}$ (first excited state). Then the points calculated in the interval $3.5 a_{0}$ to $7.0 a_{0}$ were fitted by a Morse potential (the points and fitting curves are given in Figure 1) and spectroscopic parameters together with the corresponding FC factors were determined. The FC factors were calculated by the MATHEMATiCA script for FC factors calculation between vibrational Morse oscillator states taken from Ref. 31]. The results obtained are presented in Table II

The laser cooling transition $\left({ }^{2} \Pi_{1 / 2}\left(v^{\prime}=0\right) \leftarrow\right.$ $\left.{ }^{2} \Sigma_{1 / 2}(v=0)\right)$ is found to lie around $710 \mathrm{~nm}$, and an upper state lifetime on the order of $\sim 25 \mathrm{~ns}$ is expected. With these parameters the capture range for optical molasses is $\sim 5 \mathrm{~m} / \mathrm{s}$, and the doppler limit temperature is $\sim 150 \mu \mathrm{K}$. If a precooled sample of molecules can be created, as is described in the next section, only a few thousand photons would have to be scattered per molecule to reach this temperature. At these temperatures only a very small volume in the trap is occupied, opening the possibility to perform precision measurements using the trapped molecules.

The sum of the FC factors for the ground vibrational state of the ${ }^{2} \Pi_{1 / 2}$ state and three vibrational states of ${ }^{2} \Sigma_{1 / 2}$ state is equal to 0.9999 with four digits after a point being stable in respect to variations of the fitting parameters of Morse potential (see supplementary materials for details). We would like to emphasize here, that high digital precision in calculations of $\mathrm{FC}$ factors does not mean high accuracy of the spectroscopic constants calculations. Reliable estimate of the sum of FC factors which can be used for practical purposes is expected to have two digits after a decimal point. Nevertheless both numerical results and arguments from electronic structure theory provide, in our opinion, good reasons for considering RaF for measurements of NSDPV effect and for direct laser cooling.

TABLE II: Estimated molecular spectroscopic parameters for the electronic ground state ${ }^{2} \Sigma_{1 / 2}$ and the first electronically excited ${ }^{2} \Pi_{1 / 2}$ state from FS-CCSD calculations of RaF. The dissociation energy $\tilde{D}$ was obtained as the difference between total energies of the states at the equilibrium distance $R_{e}$ and at $9.0 a_{0}$ without attempting to correct for basis set superposition errors. Fitting parameters of Morse potential with the corresponding standard errors can be found in supplementary materials. Parameter $\tilde{\omega}_{\mathrm{e}}$ is the standard harmonic vibrational wavenumber. We would like to emphasize that the accuracy of the determination of the parameters is lower (especially for $\tilde{D}$ and $\tilde{T}_{\mathrm{e}}$ ) than the number of digits reported. Nevertheless we report the results with high digital precision to provide reaper points for future calculations.

\begin{tabular}{lllll} 
& $R_{\mathrm{e}} / a_{0}$ & $\tilde{\omega}_{\mathrm{e}} / \mathrm{cm}^{-1}$ & $\tilde{D}_{\mathrm{e}} / \mathrm{cm}^{-1}$ & $\tilde{T}_{\mathrm{e}} / \mathrm{cm}^{-1}$ \\
\hline${ }^{2} \Sigma_{1 / 2}$ & 4.24 & 428 & 32104 & \\
& & & & \\
${ }^{2} \Pi_{1 / 2}$ & 4.24 & 432 & 31302 & 14012 \\
\hline
\end{tabular}

Proposed measurement strategy and sensitivity to NS$D P V$ signal The $W_{\text {a }}$ parameter can be obtained in a diatomic molecule by a measurement of the parity-violating coupling between hyperfine-rotational levels of opposite parity. An experimental procedure to do so in a molecular beam has been proposed in Ref [8], where tuning of the close-lying spin-rotational levels of opposite parity to near-degeneracy due to the Zeeman effect is used to increase the P-odd asymmetry in the measured signal. The accuracy in the determination of $W_{\mathrm{a}}$ that can be reached in such an experiment is proportional to the count rate of the molecular signal $(d N / d t)$, the interaction (coherence) time $(T)$ and the total measurement time $(\tau)$ as $\delta W_{\mathrm{a}} \sim 1 /(T \sqrt{\tau d N / d t})$.

For an interaction region of $5 \mathrm{~cm}$ and a molecular beam 
velocity of $500 \mathrm{~m} / \mathrm{s}$, the interaction time is on the order of $100 \mu \mathrm{s}$. As it has been demonstrated that cold molecules can be trapped for at least a number of seconds [32, 33], the potential gain in accuracy is 4 orders of magnitude. It is also clear that part of this gain is lost as the number of molecules will probably be low; so production and delivery of the RaF molecules to the working volume is crucial for the proposed measurements. On the other hand the high Franck-Condon overlap allows for the detection of multiple photons even from a single trapped molecule.

The two radium isotopes that are most suitable are ${ }^{213} \mathrm{Ra}$ and ${ }^{225} \mathrm{Ra}$, with lifetimes of $164 \mathrm{~s}$ and 14.9 days, respectively. Both isotopes have a nuclear spin $I=1 / 2$, which reduces the number of lasers required for lasercooling. The ${ }^{213} \mathrm{Ra}$ isotope has been successfully produced and used in experiments at the KVI 34], whereas ${ }^{225} \mathrm{Ra}$ is best obtained from a ${ }^{229} \mathrm{Th}$ source. The radium ions from the cyclotron can be implanted in a solid $\mathrm{AlF}_{3}$ target. RaF molecules can be produced from such a target by laser ablation. Similar molecules have been obtained in the rovibriational groundstate by the use of a cold buffer gas or supersonic expansion [35, 36]. These precooling methods greatly reduce the amount of photons that have to be scattered in order to reach the doppler temperature, which is a large advantage if the cooling cycle is not completely closed.

A supersonic beam can be combined with a Stark decelerator to bring the molecules to rest in the lab frame. A fraction of about $10^{-2}$ of the molecules in the supersonic expansion can be trapped. Heavy diatomic molecules such as RaF have not been decelerated to standstill yet; but a recent demonstration of an improvement to the Stark-deceleration technique [37] indicates how this could be done. The efficiency of capturing the ablated molecules in the supersonic beam is however only about $10^{-8}$ [36, 38], which is prohibitively low for RaF.

If the ablation is performed in a buffer gas cell, the cold molecules can be captured by a magnetic trap [39]. The buffer gas has to be quickly removed [40], and subsequently lasercooling could be used to bring the trapped cloud to the lower temperatures that are required for a precision measurement. A promising number of $10^{12} \mathrm{YbF}$ molecules, produced in a single laser ablation shot, has been reported [35] but it remains to be seen how many of these can eventually be trapped and used for a precision measurement.

To conclude, we have investigated the suitability of the radium fluoride ( $\mathrm{RaF}$ ) molecule to probe parity violation, which has so far not been detected in molecules. Explicit $a b$ initio calculations of the parity violating nuclear spin dependent parameter $W_{\mathrm{a}}$ and Franck-Condon factors for transitions between the electronic ground state and the lowest doublet excited state indicate that this open-shell molecule is a promising candidate for direct laser cooling and (subsequent) measurement of the nuclear anapole moment. We have also discussed possible approaches to creation, trapping and cooling of RaF molecules. In our opinion $\mathrm{RaF}$ is a valuable addition to the variety of molecules that may be used to probe parity violation, providing complimentary insight on the NSDPV effects.

Acknowledgements We are indebted to Sophie Nahrwold, Mikhail Kozlov, Klaus Jungmann and Rob Timmermans for discussions. Financial support by the Volkswagen Foundation and computer time provided by the Center for Scientific Computing (CSC) Frankfurt is gratefully acknowledged.

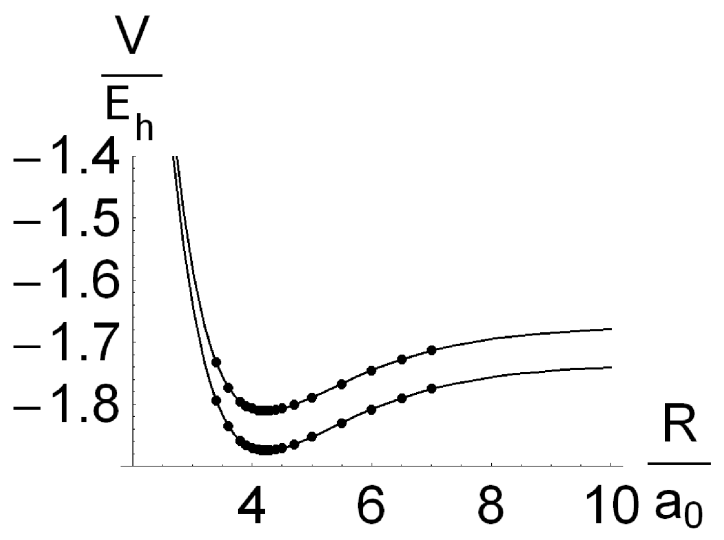

FIG. 1: Morse potential fitting of the calculated potential energy points for the two energetically lowest-lying electronic states of $\mathrm{RaF}$ - the ground electronic state is ${ }^{2} \Sigma_{1 / 2}$ and first electronically excited state is ${ }^{2} \Pi_{1 / 2}$.

* Electronic address: isaev@fias.uni-frankfurt.de

$\dagger$ Electronic address: s.hoekstra@rug.nl

$\ddagger$ Electronic address: robert.berger@tu-darmstadt.de

[1] G. Laubender and R. Berger, ChemPhysChem 4, 395 (2003).

[2] S. Nahrwold and R. Berger, J. Chem. Phys. 130, 214101 (2009).

[3] M. Kozlov and L. Labzowsky, J. Phys. B 28, 1933 (1995).

[4] B. Zel'dovich, J. Exptl. Theoret. Phys. (U.S.S.R) 33, 1531 (1957).

[5] I. Zel'dovich, Sov. Phys. JETP 6, 1184 (1958).

[6] C. S. Wood and et al, Science 275, 1759 (1997).

[7] J. S. M. Ginges and V. V. Flambaum, Phys. Rep. 397, 63 (2004).

[8] D. DeMille, S. B. Cahn, D. Murphree, D. A. Rahmlow, and M. G. Kozlov, Phys. Rev. Lett. 100, 023003 (2008).

[9] L. D. Carr, D. DeMille, R. V. Krems, and J. Ye, New J. Phys. 11, 055049 (2009).

[10] H. L. Bethlem, G. Berden, and G. Meijer, Phys. Rev. Lett. 83, 1558 (1999).

[11] H. L. Bethlem, G. Berden, F. M. H. Crompvoets, R. T. Jongma, A. J. A. van Roij, and G. Meijer, Nature 406, 491 (2000).

[12] S. Y. T. van de van de Meerakker, H. L. Bethlem, and G. Meijer, Nat. Phys. 4, 595 (2008).

[13] M. D. DiRosa, Eur. Phys. J. D 31, 395 (2004). 
[14] B. K. Stuhl, B. C. Sawyer, D. Wang, and J. Je, Phys. Rev. Lett. 101, 243002 (2008).

[15] E. S. Shuman, J. F. Barry, D. R. Glenn, and D. DeMille, Phys. Rev. Lett. 103, 223001 (2009).

[16] E. Traykov and et al, Nucl.Instrum.Meth B 266, 4532 (2008).

[17] L. W. Wansbeek, B. K. Sahoo, R. G. E. Timmermans, K. P. Jungmann, B. P. Das, and D. Mukherjee, Phys. Rev. A. 78, 050501 (2008).

[18] S. De, U. Dammalapati, K. Jungmann, and L. Willmann, Phys. Rev. A 79, 041402(R) (2009).

[19] T. A. Isaev, S. Nahrwold, and R. Berger (2010), to be published.

[20] J. L. Stuber and J. Paldus, Symmetry breaking in the independent particle model (Pergamon Press, New York, 2003), Fundamental World of Quantum Chemistry.

[21] R. Berger, N. Langermann, and C. van Wüllen, Phys. Rev. A 71, 042105 (2005).

[22] R. Berger and C. van Wüllen, J. Chem. Phys. 122, 134316 (2005).

[23] R. Ahlrichs, M. Bär, M. Häser, H. Horn, and C. Kölmel, Chem. Phys. Lett. 162, 165 (1989).

[24] M. Häser and R. Ahlrichs, J. Comput. Chem. 10, 104 (1989).

[25] C. van Wüllen, J. Chem. Phys. 109, 392 (1998).

[26] W. Liu, C. van Wüllen, F. Wang, and L. Li, J. Chem. Phys. 116, 3626 (2002).

[27] T. A. Isaev, N. S. Mosyagin, A. N. Petrov, and A. V. Titov, Phys. Rev. Lett. 95, 163004 (2005).

[28] K. P. Huber and G. Herzberg, Constants of Diatomic Molecules (Van Nostrand-Reinhold, New York, 1979).

[29] DIRAC, a relativistic ab initio electronic structure program, Release DIRAC08 (2008), written by L. Visscher, H. J. Aa. Jensen, and T. Saue, with new contributions from R. Bast, S. Dubillard, K. G. Dyall, U. Ekström, E. Eliav, T. Fleig, A. S. P. Gomes, T. U. Helgaker, J. Henriksson, M. Iliaš, Ch. R. Jacob, S. Knecht, P. Norman, J. Olsen, M. Pernpointner, K. Ruud, P. Sałek, and J. Sikkema (see http://dirac.chem.sdu.dk).

[30] L. Visscher, E. Eliav, and U. Kaldor, Journal Of Chemical Physics 115, 9720 (2001).

[31] J. C. Lopez, A. L. Rivera, Y. F. Smirnov, and A. Frank, International Journal Of Quantum Chemistry 88, 280 (2002).

[32] S. Hoekstra, J. J. Gilijamse, B. Sartakov, N. Vanhaecke, L. Scharfenberg, S. Y. T. van de van de Meerakker, and G. Meijer, Phys. Rev. Lett. 98, 133001 (2007).

[33] N. Vanhaecke and O. Dulieu, Mol. Phys. 105, 1723 (2007).

[34] O. O. Versolato, G. S. Giri, L. W. Wansbeek, J. E. van den Berg, D. J. van der Hoek, K. Jungmann, W. L. Kruithof, C. J. G. Onderwater, B. K. Sahoo, B. Santra, et al., Phys. Rev. A 82, 010501 (2010).

[35] M. R. Tarbutt, J. J. Hudson, B. E. Sauer, and E. A. Hinds, Faraday Discuss. 142, 37 (2009).

[36] M. R. Tarbutt, J. J. Hudson, B. E. Sauer, E. A. Hinds, V. A. Ryzhov, V. L. Ryabov, and V. F. Ezhov, J. Phys. B-At. Mol. Opt. 35, 5013 (2002).

[37] A. Osterwalder, S. A. Meek, G. Hammer, H. Haak, and G. Meijer, Phys. Rev. A 81, 51401 (2010).

[38] P. R. Willmott and J. R. Huber, Rev. Mod. Phys. 72, 315 (2000).

[39] J. D. Weinstein, R. Decarvalho, T. Guillet, B. Friedrich, and J. M. Doyle, Nature 395, 148 (1998).

[40] J. Harris, R. Michniak, S. Nguyen, N. Brahms, W. Ketterle, and J. Doyle, Europhys. Lett. 67, 198 (2004). 